\title{
Corrigenda \\ GLYX-I3, a NMDA Receptor Glycine-Site Functional Partial Agonist, Induces Antidepressant-Like Effects Without ketamine-like Side Effects
}

\author{
Jeffrey Burgdorf, Xiao-lei Zhang, Katherine L Nicholson, Robert L Balster, J David Leander, \\ Patric K Stanton, Amanda L Gross, Roger A Kroes and Joseph R Moskal
}

Neuropsychopharmacology (2013) 37, 1374; doi:10.1038/npp.2013.42

Correction to: Neuropsychopharmacology advance online publication, 30 January 2013; doi:10.1038/npp.2013.42

In this article, there are errors in page 2, right column, fifth and sixth lines in the 'Drugs' section: the sentence should read 'Scrambled GLYX-13 (proline-threonine-prolinethreonine- $\mathrm{NH}_{2}$ ) was purchased from Bachem (USA).' Also in figure 1 legend, the second line should read 'scrambled GLYX-13 (PTPT- $\mathrm{NH}_{2} ; 3$ mg/kg, IV)'.

\section{fosB-Null Mice Display Impaired Adult Hippocampal Neurogenesis and Spontaneous Epilepsy with Depressive Behavior}

Noriko Yutsudo, Takashi Kamada, Kosuke Kajitani, Hiroko Nomaru, Atsuhisa Katogi, Yoko H Ohnishi, Yoshinori N Ohnishi, Kei-ichiro Takase, Kunihiko Sakumi, Hiroshi Shigeto and Yusaku Nakabeppu

Neuropsychopharmacology (2013) 38, 1374-1375; doi:10.1038/npp.2013.56

Correction to: Neuropsychopharmacology (2013) 38, 895-906; doi:10.1038/npp.2012.260; published online 16 January 2013

In this article, the parenthetical text in lines 10 and 11 of the Acknowledgments should read '(Cell-fate Decision: Function and Dysfunction in Homeostasis).'

In page 899, left column, line 17 should read 'The remainder of BrdU-positive cells mostly expressed either DCX or NeuN.'

Corrections were made in Figure 4c; a revised version with caption is provided in the next page.
In page 904, left column, line 35 should read ' $f o s B$-null mice exhibited abnormalities in hippocampal structures, which were similar to hippocampal sclerosis observed in the brains of rodent models and human patients with epilepsy (Fahrner et al, 2007).'

In page 904, right column, line 36 should read 'The 10 genes listed in Table 1 are expressed in hippocampal neurons.'

In page 904, right column, line 39 should read 'As VGF, TRH, GAL, DLK1, and PENK are secretory molecules.' 